\title{
Socioeconomic deprivation and health in Glasgow and the west of Scotland - a study of cancer incidence among male residents of hostels for the single homeless
}

\author{
Douglas W Lamont, Fiona M Toal, Maureen Crawford
}

\begin{abstract}
Study objective-To determine the incidence of cancer among homeless men in Glasgow.

Design-Descriptive study of cancer incidence in a defined, though individually unidentifiable, population cohort.

Setting-Glasgow and the West of Scotland Region.

Participants-Male residents of 10 hostels for the single homeless in Glasgow, open for all or part of the period 1975-93. Estimated total man-years of risk 21820. Main results-After adjusting for age and socioeconomic deprivation, the proportional incidence ratio (PIR) of tumours of the oral cavity and pharynx in hostel residents was over twice what would be expected in the male population as a whole (PIR 2.37, 95\% CI 1.41, 4.00). Cancers of the oesophagus and larynx were also overrepresented (PIR 1.63 and 1.74 respectively). Estimated age standardised incidence ratios were greater than would be expected for the most socioeconomically deprived areas of the west of Scotland for tumours of the oral cavity and pharynx, larynx, oesophagus, and lung (2.39, 1.87, 1.61, and 1.23 respectively). Conclusions-The incidence of many cancers is known to be higher in lower socioeconomic groups. Within the lowest deprivation category, there is evidence from this study for a further excess risk among homeless men for cancers of the oral cavity and pharynx, oesophagus, larynx, and lung. Improvements in general health care are urgently needed for this particularly vulnerable section of the population.
\end{abstract}

$(\mathcal{E}$ Epidemiol Community Health 1997;51:668-671)

Department, Greater Glasgow Health Board, Glasgow G3 8YY F M Toal

Dental Public Health, Department of Public Health, Newcastle and North Tyneside Health Authority, Newcastle upon Tyne NE6 4PF M Crawford

Correspondence to: Dr D Lamont.

Accepted for publication February 1997 recent work by McLoone and Boddy ${ }^{9}$ and McCarron, Davey Smith, and Womersley ${ }^{10}$ has shown that a widening in mortality differentials has taken place since 1980 between affluent and deprived areas, both in Scotland as a whole and in greater Glasgow. These changes have occurred despite an overall reduction in deaths among those aged $0-64$ over this period. Increasing mortality differentials have also been reported from the north of England. ${ }^{11}$

A study of the health effects of deprivation at both an individual and area level has shown very high correlations between personal disease risks and the variables used to characterise areas of residence as affluent or deprived. ${ }^{12}$ There was no evidence for a location-specific ecological effect - similarly disadvantaged individuals had higher mortality risks wherever they lived. It is necessary therefore to target individuals, or particularly disadvantaged groups of people, in any intervention to reduce the health consequences of socioeconomic deprivation.

We report here an investigation in greater Glasgow into the incidence over the last 20 years of 10 different types of cancer in what is arguably one of the most deprived sections of the community - male residents of hostels for the single homeless. Single homeless people have a higher risk of death and disease than comparable groups in the general population, with oral, gastrointestinal, and respiratory conditions figuring prominently among nontraumatic causes of excess mortality and morbidity. ${ }^{13}$ Some may be unwell before becoming homeless-ill health often limits employment opportunities and income, while mental illness may also lead to loss of accommodation. ${ }^{13}$

\section{Methods}

The population for this study comprised male residents of 10 hostels for the single homeless in Glasgow open for all or part of the period 1975-93. While cancer registration data are limited in the extent to which they can describe the socioeconomic circumstances of individual patients, availability of the full unit postcode permits identification and analysis of institutional populations, provided the postcode is unique to the address required. The Glasgow hostels chosen were those which had a unique unit postcode from 1975-93. All 10 hostels were located in areas classified as being among 
the most socioeconomically deprived in the west of Scotland (Carstairs ${ }^{8}$ deprivation category 7).

All cases of cancer diagnosed between 1975 and 1993 in persons resident at hostel postcodes were identified from the records of the West of Scotland Cancer Registry. Cancer registration records did not enable us to identify officially homeless persons (mainly pregnant women and families with children ${ }^{13}$ ) or those sharing accommodation or otherwise inadequately housed. Those who were sleeping rough and others with no address of their own were also excluded. There were only 20 registrations in the 19 year period with an NF1 (no fixed abode) postcode and 2 with addresses listed as care of an acute (rather than long stay) hospital.

The proportional incidence of 10 cancers and groups of cancers in Glasgow hostel residents was compared with that in the adult male population of the west of Scotland as a whole, adjusting for age and socioeconomic deprivation. No information was available on either the average level of occupancy of each hostel during the study period or on the age structure of the hostel population. All hostels were assumed therefore to have been fully occupied over the study period and the total number of man-years of risk was estimated to be 21820 (calculated by multiplying the total number of bed spaces in each hostel by the number of years in operation). Crude and indirectly age standardised cancer incidence rates were estimated using this total population and an age structure derived from questionnaire survey of homeless men in Leeds. ${ }^{14}$

Estimated standardised incidence ratios for hostel residents compared to all men in the west of Scotland living in areas within the same Carstairs ${ }^{8}$ deprivation category were also calculated. Since standardised incidence ratios, assuming no change in age structure, will increase in direct proportion to a reduction in person years of risk, a sensitivity analysis was carried out to examine the effect of lower rates of occupancy on these ratios and their statistical significance. Results are presented assuming both full occupation of the 10 hostels (chosen to give the most conservative estimate of cancer incidence in the study population) and partial occupation at a point $(75 \%)$ below which there was no change in the statistical sig-
KEY POINTS

- The incidence of many cancers is higher in lower socioeconomic groups.

- Male residents of hostels for the single homeless seem to be at even greater risk than other deprived populations for cancers of the oral cavity and pharynx, oesophagus, larynx, and lung.

- Improvements in general health service provision are urgently required for this especially vulnerable section of the community.

nificance of any of the incidence ratios until a much lower level of occupancy had been reached.

\section{Results}

The proportional incidence of tumours of the oral cavity and pharynx in hostel residents was, after adjusting for age and socioeconomic deprivation, over twice what would be expected in the adult male population as a whole (proportional incidence ratio ((PIR) $2.37,95 \% \mathrm{CI}$ $1.41,4.00$ ) (table 1). Cancers of the oesophagus and larynx were also over-represented (PIR 1.63 and 1.74 respectively), while colorectal and non-melanoma skin cancers accounted for significantly fewer cancer cases among hostel residents (PIR 0.50 and 0.51 respectively). Stomach cancers were also under-represented (PIR 0.74). Lung tumours were slightly more common (PIR 1.23).

Estimated crude cancer incidence among hostel residents for all sites combined (table 2) was considerably higher than for men of similar socioeconomic status in the population as a whole (1169 and 762 per 100000 respectively), but slightly lower when age standardised (729 per 100 000). Estimated site specific age standardised incidence ratios (SIR), however, were greater than would be expected for all men living in the most socioeconomically deprived areas of the west of Scotland for tumours of the oral cavity and pharynx, larynx, oesophagus, and lung (SIR 2.39, 1.87, 1.61, and 1.23). These results were statistically significant for oropharyngeal and lung cancer $(p<0.05$, two tailed), but not for tumours of the oesophagus and larynx. The excess of cancers of the oral cavity and pharynx remained

Table 1 Proportional incidence ratio (PIR) for selected cancers in Glasgow hostel residents in 1975-93 (males aged 15 and over, Carstairs deprivation category 7)

\begin{tabular}{llll}
\hline Tumour site & Observed cases & Expected cases & $P I R(95 \%$ CI $)$ \\
\hline Oral cavity pharynx + & 13 & 5.48 & $2.37(1.41,4.00)$ \\
Oesophagus & 11 & 6.73 & $1.63(0.92,2.91)$ \\
Larynx & 10 & 5.76 & $1.74(0.95,3.19)$ \\
Lung & 125 & 101.59 & $1.23(1.09,1.39)$ \\
Stomach & 12 & 16.26 & $0.74(0.43,1.28)$ \\
Colon, rectum & 12 & 23.84 & $0.50(0.29,0.87)$ \\
Skin (excluding melanoma) & 11 & 21.78 & $0.51(0.28,0.90)$ \\
Prostate & 10 & 13.58 & $0.74(0.40,1.34)$ \\
Bladder & 9 & 14.51 & $0.62(0.33,1.18)$ \\
Lymphatic, haematopoieticł & 11 & 9.42 & $1.17(0.66,2.07)$ \\
Other sites & 31 & 36.05 & $0.86(0.62,1.19)$ \\
All sites (ICD9 140-208) & 255 & 255.00 & 1.00 \\
\hline
\end{tabular}

* If proportional distribution was the same as in the west of Scotland as a whole, adjusted for age and socioeconomic deprivation. $\dagger$ Excluding tumours of the salivary gland, nasopharynx and oral cavity unspecified.

‡ Lymphoma, multiple myeloma and leukaemia. 
Table 2 Estimated crude (CR) and age standardised (ASR) incidence rates per 100000 for selected cancers in Glasgow hostel residents $1975-93$ (males aged 15 and over, Carstairs deprivation category 7)

\begin{tabular}{|c|c|c|c|c|c|c|}
\hline \multirow[b]{2}{*}{ Tumour site } & \multicolumn{2}{|c|}{$\begin{array}{l}\text { Glasgow hostel } \\
\text { residents }\end{array}$} & \multirow{2}{*}{$\begin{array}{l}\begin{array}{l}\text { West of } \\
\text { Scotland }\end{array} \\
C R\end{array}$} & \multicolumn{2}{|c|}{ Standardised incidence ratio (SIR) $(95 \% \mathrm{CI})$} & \multirow{2}{*}{$\begin{array}{l}\text { Occupancy threshold for a } \\
\text { statistically significant } \\
\text { SIR }>1.00(\%)\end{array}$} \\
\hline & $\overline{C R}$ & $A S R$ & & $100 \%$ Occupancy & $75 \%$ Occupancy & \\
\hline Oral cavity, pharynx ${ }^{\star}$ & 59.6 & 38.8 & 16.2 & $2.39(1.27,4.09)$ & $3.19(1.70,5.46)$ & 100 \\
\hline Oesophagus & 50.4 & 31.9 & 19.9 & $1.61(0.80,2.88)$ & $2.14(1.07,3.84)$ & 79 \\
\hline Larynx & 45.8 & 30.6 & 16.4 & $1.87(0.90,3.43)$ & $2.49(1.19,4.58)$ & 89 \\
\hline Lung & 572.9 & 358.6 & 291.8 & $1.23(1.03,1.42)$ & $1.64(1.41,1.86)$ & 100 \\
\hline Stomach & 55.0 & 34.0 & 47.7 & $0.71(0.37,1.25)$ & $0.95(0.49,1.67)$ & 36 \\
\hline Colon, rectum & 55.0 & 33.4 & 71.9 & $0.47(0.24,0.81)$ & $0.62(0.32,1.09)$ & 24 \\
\hline Skin (excluding melanoma) & 50.4 & 30.7 & 65.8 & $0.47(0.23,0.84)$ & $0.62(0.31,1.11)$ & 23 \\
\hline Prostate & 45.8 & 25.4 & 41.3 & $0.62(0.30,1.13)$ & $0.82(0.39,1.51)$ & 29 \\
\hline Bladder & 41.2 & 25.4 & 43.0 & $0.59(0.27,1.12)$ & $0.79(0.36,1.49)$ & 26 \\
\hline Lymphatic, haematopoietic $\dagger$ & 50.4 & 33.6 & 32.3 & $1.04(0.52,1.86)$ & $1.38(0.69,2.48)$ & 51 \\
\hline Other sites & 142.1 & 91.9 & 115.4 & $0.80(0.54,1.13)$ & $1.06(0.72,1.51)$ & 54 \\
\hline All sites (ICD9 140-208) & 1168.7 & 729.4 & 761.6 & $0.96(0.84,1.08)$ & $1.28(1.14,1.42)$ & 84 \\
\hline
\end{tabular}

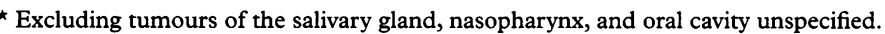

†Lymphoma, multiple myeloma, and leukaemia.

Table 3 Cancer incidence in relation to socioeconomic deprivation west of Scotland 1985-94

Standardised incidence ratios (West of Scotland $=100$ )

\begin{tabular}{llll}
\hline $\begin{array}{l}\text { Cancers with above average incidence in } \\
\text { affluent areas (Carstairs deprivation } \\
\text { categories 1,2) }\end{array}$ & & $\begin{array}{l}\text { Cancers with above average incidence } \\
\text { in deprived areas (Carstairs } \\
\text { deprivation categories 6,7) }\end{array}$ \\
\hline Melanoma & 139 & Larynx & \\
Endometrium & 119 & Lung & 149 \\
Ovary & 111 & Lip, oral cavity and pharynx & 141 \\
Female breast & 108 & Cervix & 135 \\
Non-melanoma skin & 105 & Stomach & 128 \\
& & Oesophagus & 119 \\
& & Pancreas & 112 \\
& & Bladder & 111 \\
& & Colorectal & 105
\end{tabular}

Sites for which the standardised incidence ratio was not significantly different from 100 are excluded.

significant after correction for multiple testing (adjusted $\mathrm{p}=0.034$, one-tailed). When a lower level of occupancy of $75 \%$ was assumed, however, standardised incidence ratios for these sites increased to $3.19,2.49,2.14$, and 1.64 respectively, and all four results were statistically significant (table 2). A further $20 \%$ reduction in the average occupancy of each hostel would have been required for a significant excess in incidence to be recorded for any other tumour site.

\section{Discussion}

While high proportional incidence ratios do not necessarily imply excess risk, significantly higher proportions of tumours of the oral cavity and pharynx among the homeless suggest some scope for intervention via targeted dental health checks. Oral examination by dental practitioners has already been suggested as a possible method for the early detection of asymptomatic small lesions, ${ }^{15}$ and this could be extended to the community dental service.

Considerable practical and ethical difficulties regarding stage at presentation and follow up would have to be overcome, however, for such action to be both possible and successful. Large lesions are also frequently asymptomatic ${ }^{15}$; the sensitivity and specificity of oral examination as a screening strategy is not yet known for a UK population ${ }^{15}$; and both treatment acceptance and effectiveness are likely to be poor in such a highly mobile and physically vulnerable population. Problems associated with follow up cannot be underestimated-with the exception of those sharing accommodation or otherwise inadequately housed, direct access hostel residents are the most readily contactable group of the unofficially homeless population, and those sleeping rough the least.

The age structure of the Glasgow hostel population was estimated using results from a survey carried out elsewhere in Britain. No firm conclusions can be drawn from our data regarding higher than expected age standardised incidence rates until local surveys have been carried out. Similarly, in the absence of information on levels of occupancy, each hostel was assumed to be fully occupied throughout the study period, although we have shown that this would tend to bias rates downwards. An average occupancy of $75 \%$ would have increased incidence rates sufficient to result in statistically significant excesses of tumours of the oesophagus and larynx as well as of those of the oral cavity and lung. Conversely, colorectal and non-melanoma skin cancers would no longer have been significantly under represented.

The incidence of many cancers is known to be higher in lower socioeconomic groups (table 3). Within the lowest of these (Carstairs deprivation category 7) there does seem to be some evidence from this study for a further excess risk among homeless men for cancers of the oral cavity and pharynx, oesophagus, larynx, and lung. While smoking and excessive alcohol consumption are the main risk factors associated with these malignancies, dietary deficiencies in the form of iron depletion and low levels of vitamin $A$ and $\beta$-carotene have also been implicated in the aetiology of tumours of the oral cavity, pharynx, and oesophagus. ${ }^{16}{ }^{17}$ The legacy of an earlier period of poor housing, moreover, in the form of lung damage in childhood sustained in part as a result of damp and overcrowded living conditions in the 1920's and 30 's, has recently been suggested as a possible explanation for the particularly high incidence of lung cancer in the west of Scotland. ${ }^{18}$

Stomach cancers were under represented in the Glasgow hostel population, despite a strong association with deprivation (table 3 ). This result, however, was not statistically significan 
at any level of occupation and the $95 \%$ confidence interval assuming $75 \%$ occupancy included the west of Scotland standardised incidence ratio for deprivation categories 6 and 7. Although colorectal and non-melanoma skin cancers would have been significantly under represented if each hostel had been fully occupied, colorectal cancer is only slightly more common among residents of deprived areas than in the population as a whole and there is no significant excess of non-melanoma skin cancer (table 3). Improvements in general health care are urgently needed for this particularly vulnerable section of the population. Suggestions made in a recent report for the Royal College of Physicians of London included the funding of special GP practices for homeless people. ${ }^{13}$ Ultimately, however, the underlying social problems of poverty and poor housing conditions in general, and homelessness in particular, require to be addressed.

An earlier version of this paper was presented at the 4th Annual Conference of the United Kingdom Association of Cancer Registries, Titchfield, Hampshire, November 1995. We are gratefu to Jan Richardson and Lisa Allsopp for secretarial assistance, and to Dr Charles Gillis, Director of the West of Scotland Cancer Surveillance Unit, for access to cancer registry data. Conflicts of interest: none.

1 Davey Smith G, Bartley M, Blane D. The Black report on socioeconomic inequalities in health 10 years on. $B M F$ 1990;301:373-7.
2 Davey Smith G, Egger $M$. Socioeconomic differentials in wealth and health: the legacy of the Thatcher years. $B M F$ 1993;307:1085-6.

3 Power C. Health and social inequality in Europe. BMF 1994;308:1153-6.

4 Mackenbach JP. Socioeconomic inequalities in health in the Netherlands: impact of a five-year research programme. BMF 1994;309:1487-91.

5 Davey Smith G, Morris J. Increasing inequalities in the health of the nation. $B M \mathcal{F} 1994 ; 309: 1453-4$.

6 Judge K. Beyond health care. BMf 1994;309:1454-5.

7 Wilkinson RG. Divided we fall. BMF 1994;308:1113-4

8 Carstairs V, Morris R. Deprivation and health in Scotland. Aberdeen: Aberdeen University Press, 1991.

9 McLoone P, Boddy F. Deprivation and mortality in Scotland, 1981 and 1991. BMF 1994;309:1465-70.

10 McCarron PG, Davey Smith G, Womersley JJ. Deprivation and mortality in Glasgow: changes from 1980 to 1992 BM7 1994;309:1481-2.

11 Phillimore P, Beattie A, Townsend P. Widening inequality of health in northern England, 1981-91. BMF 1994;308: $1125-8$.

12 Sloggett A, Joshi H. Higher mortality in deprived areas: community or personal disadvantage? $B M F 1994 ; 309$ $1470-4$

13 Connolly J, Crown J, eds. Homelessness and ill health. London: Royal College of Physicians, 1994

14 Blackmore T, Williams SA, Prendergast MJ, Pope JEC. The dental health of single male hostel dwellers in Leeds. Community Dental Health 1995;12:104-9.

15 United Kingdom working group on screening for oral cancer and pre-cancer. Conclusions and recommendations. Community Dental Health 1993;10(suppl):87-9.

16 Binnie WH. Risk factors/markers in low incidence areas. In Johnson NW, ed. Risk markers for oral diseases Vol.2 Oral cancer: detection of patients and lesions at risk. Cambridge: University Press, 1991; 64-87.

17 Negri E, La Vecchia C, Franceschi S, Decarli A, Bruzzi P. Attributable risks for oesophageal cancer in northern Italy. Eur $\mathcal{F}$ Cancer 1992;28A:1167-71.

18 Hole DJ, Gillis CR, Watt GM. Does early respiratory environment influence lung cancer risk? Evidence from studies in the west of Scotland. F Epidemiol Community Health 1995;49:548-9. 